transport to NAD at the expense of $\mathrm{H}_{2}$ would be inhibited to the same extent as the succinate-linked reaction. However, since the $\mathrm{H}_{2}$-linked reduction of cytochrome $\mathrm{c}$ is only weakly inhibited at low concentrations of antimycin $\mathrm{A}$, the noncyclic system can operate under these conditions. It is suggested that the ascorbate-DCPIP-linked NAD-photoreduc- tion by $R h$. rubrum chromatophores also involves a light-induced noncyclic electron transport.

The author is indebted to Prof. Dr. H. G. Schlegel for his encouragement to this work and to Dr. K.-D. Lippert for stimulating discussions. The work was supported by the Deutsche Forschungsgemeinschaft.

\title{
Polarographische Messung kritischer Sauerstoffdrucke bei Herzmuskelsarkosomen
}

\author{
H. Glossmann * und M. Frimmer \\ Institut für Pharmakologie und Toxikologie an der Veterinärmedizinischen Fakultät \\ der Justus Liebig-Universität Gießen (Direktor : Prof. Dr. med. M. Frimmer) \\ (Z. Naturforschg. 24 b, 76-79 [1969] ; eingegangen am 20. Juni 1968)
}

\begin{abstract}
Mit Hilfe der Ganzglas-Platinelektrode nach Gleichmans und Lübbers wurde der kritische $\mathrm{O}_{2}$ Druck bei stark verdünnten Sarkosomensuspensionen in Tris-Puffer bei $37^{\circ} \mathrm{C}$ mit $2,14 \pm 0,87$ Torr ermittelt. Die Abhängigkeit der kritischen $\mathrm{O}_{2}$-Drucke vom Suspensionsmilieu (Tris- und Phosphatpuffer) sowie die Beeinflussung der Meßergebnisse durch Hämoglobin oder Myoglobin wurden untersucht.
\end{abstract}

Die Kenntnis der kritischen $\mathrm{O}_{2}$-Drucke in Zellen bzw. Mitochondrien ist für zahlreiche Probleme der Physiologie und Pharmakologie von großem Interesse (s. Thews ${ }^{2,3}$ ). Die 1955 von Bänder und $\mathrm{KIESE}^{4}$ mit einem schnell rotierenden $\mathrm{W}$ a r b u r g . Manometer gemessenen kritischen Drucke bei Lebermitochondrien betrugen bei $37^{\circ} \mathrm{C} 8-9$ Torr. In letzter Zeit sind jedoch Zweifel aufgetaucht, ob diese Werte zutreffen (LüBbers und Kessler ${ }^{5}$ ). Bei der manometrischen Bestimmung spielt die Diffusion des $\mathrm{O}_{2}$ von der Gasphase in das Suspensionsmedium eine limitierende Rolle. Da ferner die an Lebermitochondrien gemessenen Werte nicht ohne weiteres auf die Verhältnisse am Herzen übertragen werden können, haben wir die Atmung von Herzmuskelsarkosomen bei niedrigen $\mathrm{O}_{2}$-Drucken unter verschiedenen Versuchsbedingungen gemessen.

* Die Ergebnisse dieser Arbeit sind Teil einer Dissertation (Med. Fakultät der Justus Liebig-Universität Gießen) .

1 U. Gleichmann u. D. W. Lübbers, Pflügers Arch. ges. Physiol. Menschen Tiere 271, 431 [1960].

2 G. Thews, Pflügers Arch. ges. Physiol. Menschen Tiere 271, $197[1960]$.

3 G. Thews, Pflügers Arch. ges. Physiol. Menschen Tiere 276, $166[1962]$.

\section{Experimenteller Teil}

Me thoden: Sarkosomen wurden aus Herzen von etwa $200 \mathrm{~g}$ schweren Wistarratten unter Verwendung von Nargase gewonnen. (Ausführliche Darstellung s. Hegner und Glossmann ${ }^{6}$.) Die von Gleichmann und Lüвbers ${ }^{1}$ ursprünglich für die Messung von $\mathrm{O}_{2}$-Drukken im Blute entwickelte polarographische Methode wurde für die Messung an Mitochondrien adaptiert (s. Hegner und Glossmann ${ }^{6}$ ).

\section{Ergebnisse}

Die $\mathrm{O}_{2}$-Aufnahme von Herzmuskelsarkosomen wurde bei $22,5{ }^{\circ} \mathrm{C}$, bei $30{ }^{\circ} \mathrm{C}$ und bei $37{ }^{\circ} \mathrm{C}$ gemes. sen (Abb. 1). Bei $22,5^{\circ} \mathrm{C}$ bestand eine gute Proportionalität zwischen $\mathrm{O}_{2}$-Aufnahme und eingesetzter Sarkosomenmenge (mg Protein). Bei $30{ }^{\circ} \mathrm{C}$ und $37^{\circ} \mathrm{C}$ war diese Proportionalität weniger ausge-

4 A. Bänder u. M. Kiese, Arch. exp. Pathol. Pharmakol. 224. 312 [1955].

5 D. W. Lübbers u. M. Kessler, Pflügers Arch. ges. Physiol. Menschen Tiere 281, 50 [1964].

${ }^{6}$ D. Hegner u. H. Glossmann, Z. Naturforschg. 20 b. 234 [1965] 

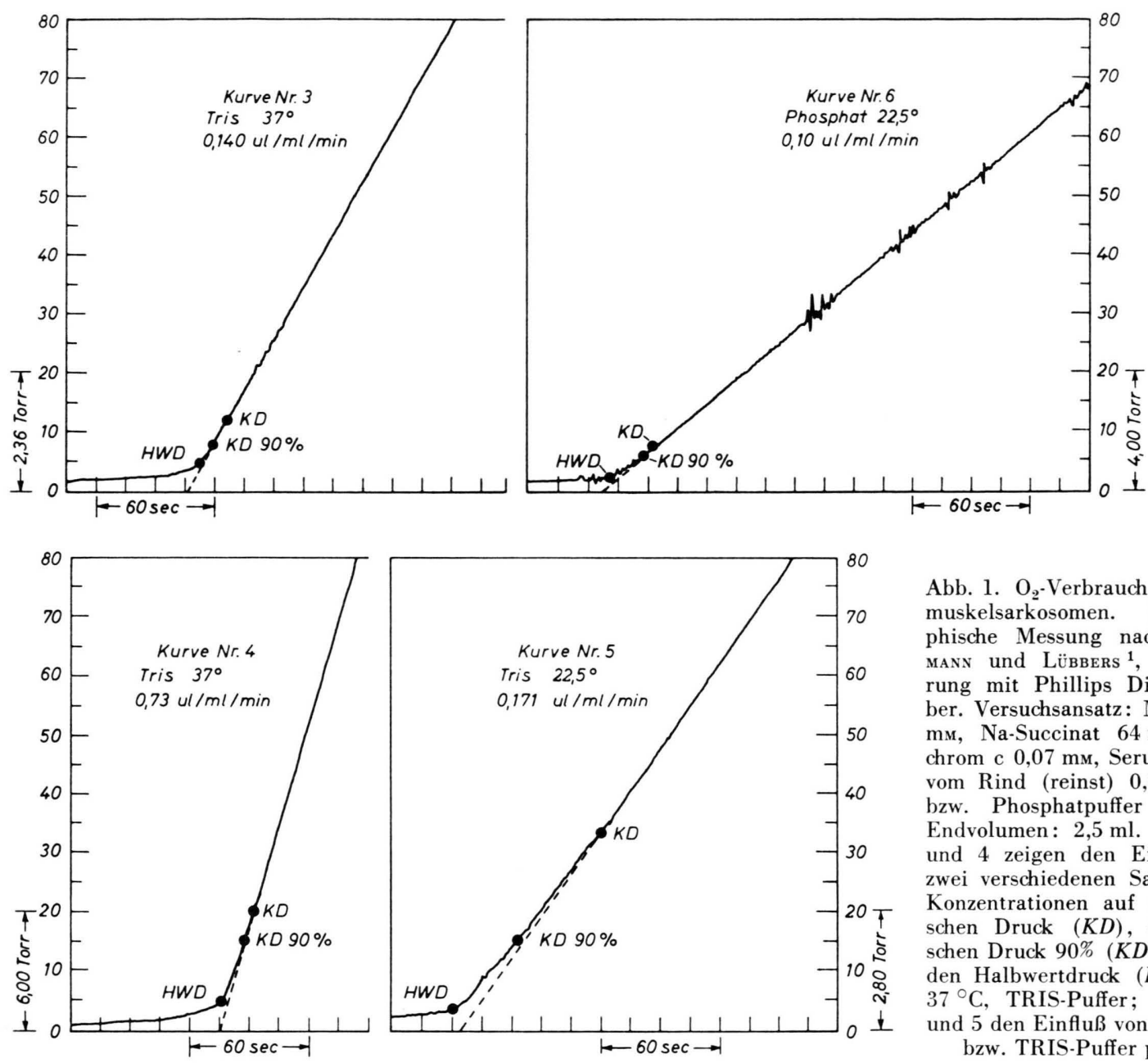

Abb. 1. $\mathrm{O}_{2}$-Verbrauch von Herzmuskelsarkosomen. Polarographische Messung nach GLEICHManN und LübBers ${ }^{1}$, Registrierung mit Phillips Direktschreiber. Versuchsansatz: $\mathrm{MgSO}_{4} 2,5$ тм, Na-Succinat $64 \mathrm{~mm}$, Cytochrom c $0,07 \mathrm{~mm}$, Serumalbumin vom Rind (reinst) 0,5\%, TRISbzw. Phosphatpuffer pH 7,4. Endvolumen: 2,5 ml. Kurven 3 und 4 zeigen den Einfluß von zwei verschiedenen SarkosomenKonzentrationen auf den Kritischen Druck $(K D)$, den Kritischen Druck $90 \%(K D$ 90\%) und den Halbwertdruck $(H W D)$ bei $37^{\circ} \mathrm{C}$, TRIS-Puffer; Kurven 6 und 5 den Einfluß von Phosphatbzw. TRIS-Puffer pH 7,4 .

\begin{tabular}{ccllllr}
\hline $\begin{array}{c}\text { Sarkosomenkonzen- } \\
\text { tration mg Protein } / \\
\text { Ansatz }\end{array}$ & $\begin{array}{c}T \\
{\left[{ }^{\circ} \mathrm{C}\right]}\end{array}$ & Puffer & $v_{\max }$ & $K D$ & $K D_{90 \%}$ & $H W D$ \\
\hline 0,1 & 22,5 & Tris & 0,098 & 6,9 & 2,25 & 0,51 \\
0,1 & 22,5 & Tris & 0,090 & 6,8 & 2,45 & 0,51 \\
0,1 & 22,5 & Phosphat & 0,600 & 4,1 & 3,70 & 1,04 \\
0,03 & 22,5 & Phosphat & 0,199 & 1,64 & 1,52 & 0,80 \\
0,015 & 22,5 & Phosphat & 0,100 & 1,44 & 1,35 & 0,45 \\
0,02 & 30,0 & Tris & 0,088 & 3,8 & 1,70 & 0,48 \\
0,02 & 30,0 & Phosphat & 0,340 & 2,35 & 2,25 & 0,85 \\
0,006 & 30,0 & Phosphat & 0,103 & 0,67 & 0,63 & 0,33 \\
0,003 & 30,0 & Phosphat & 0,066 & 0,67 & 0,63 & 0,30 \\
\hline
\end{tabular}

Tab. 1 a. $\mathrm{O}_{2}$-Verbrauch und kritische $\mathrm{O}_{2}$-Drucke von Herzmuskelsarkosomen bei Messung in Tris- und Phosphatpuffer. $v_{\mathrm{max}}$ in $\mu \mathrm{l} \mathrm{O}_{2} / \mathrm{ml} / \mathrm{min}, K D=$ kritischer $\mathrm{O}_{2}$-Druck (Torr), $K D_{90}=v=90 \% v_{\max }$ (Torr), $H W D=$ Halbwertdruck $=v=0,5 \cdot v_{\max }$ (Torr), Tris=Tris-Puffer 0,15-m., pH 7,4, Phosphat=Phosphatpuffer 0,15-m., pH 7,4.

\begin{tabular}{cccccl}
\hline$T$ & Puffer & $K D$ & & $K D_{90 \%}$ & $H W D$ \\
\hline 22,5 & Tris & $5,36 \pm 0,74$ & $(34)$ & $2,25 \pm 0,37$ & $0,47 \pm 0,07$ \\
30,0 & Tris & $3,48 \pm 1,07$ & $(20)$ & $1,58 \pm 0,30$ & $0,42 \pm 0,29$ \\
37,0 & Tris & $2,14 \pm 0,87$ & $(21)$ & $1,17 \pm 0,37$ & $0,52 \pm 0,07$ \\
\hline
\end{tabular}

Tab. 1 b. Mittelwerte der kritischen und Halbwertdrucke (in Klammern Zahl der Versuche). 
prägt: Oberhalb einer bestimmten SarkosomenKonzentration war die gemessene Atmung kleiner als erwartet. Das unerwartete Ergebnis dieser Untersuchungen war, daß mit steigender Partikelkonzentration die Halbwertsdrucke und kritischen $\mathrm{O}_{2}$ Drucke zunächst konstant blieben und dann anstiegen. Wurden die Sarkosomen-Konzentrationen in den Versuchsansätzen niedrig gehalten, so waren die gewonnenen Meßergebnisse gut reproduzierbar (Tab. 1 b).

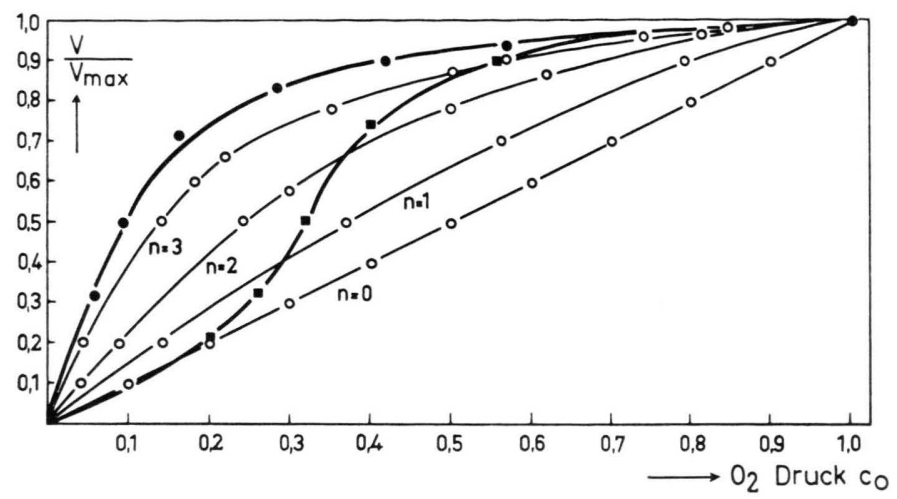

Abb. 2 a. Verhalten der Atmung unterhalb der Kritischen $\mathrm{O}_{2}$-Konzentration, die gleich 1 gesetzt wurde. Herzmuskelsarkosomen, TRIS-Puffer, $37{ }^{\circ} \mathrm{C}$; $\bullet$ Herzmuskelsarkosomen, TRIS-Puffer, $22,5^{\circ} \mathrm{C}$; 0 theoretische Werte für $v / v_{\max }$ atmender kugliger Teilchen in Abhängigkeit von $n$ (berechnet nach Gleichung 3). Für $n=0$ ist $D_{\mathrm{i}} \infty$; für $n=3$ ist $h \infty$.

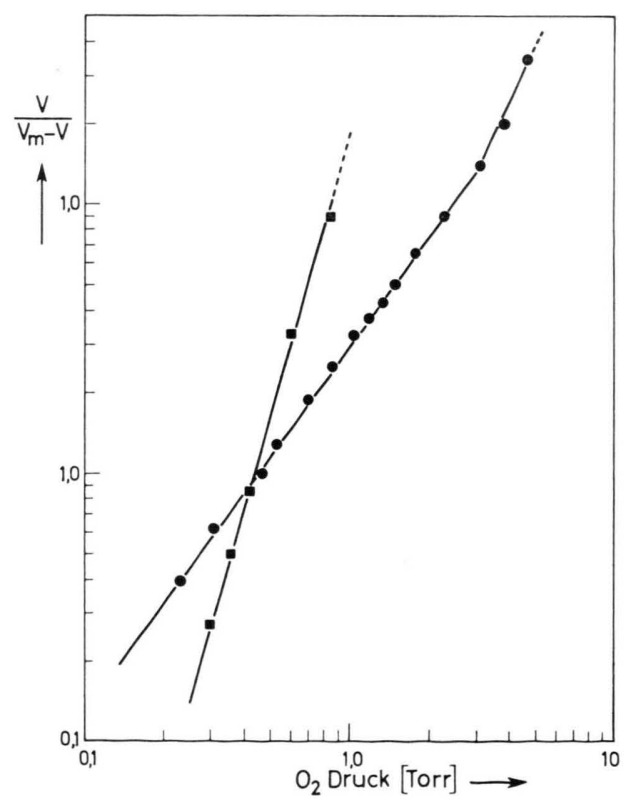

Abb. 2 b. Werte der Abb. 2 a im doppelt logarithmischen Bezugssystem aufgetragen. Herzmuskelsarkosomen, $37^{\circ} \mathrm{C}$, TRIS-Puffer; $\bullet$ Herzmuskelsarkosomen, $22,5^{\circ} \mathrm{C}$, TRIS-Puffer.
In der Tab. 1 a sind die Ergebnisse vergleichender Messungen in Tris- und Phosphatpuffer wiedergegeben. In Phosphatpuffer fanden wir höheren $\mathrm{O}_{2}$ Verbrauch und niedrigere kritische $\mathrm{O}_{2}$-Drucke als in Tris-Puffer.

Bei $22,5{ }^{\circ} \mathrm{C}$ ergab die Messung der $\mathrm{O}_{2}$-Aufnahme im Bereich unterhalb des kritischen $\mathrm{O}_{2}$-Druckes eine Kurve, die eine Michaelis-Menten-Kinetik vermuten läßt. Dagegen wurde bei $37^{\circ} \mathrm{C}$ ein sigmoidförmiger Kurvenverlauf festgestellt. Trägt man $\log \frac{v}{v_{\max }-v}$ gegen $\log \mathrm{pO}_{2}$ auf, so ergibt sich in beiden Fällen für den Bereich von $10-90 \% v_{\max }$ eine Gerade, die bei $22,5{ }^{\circ} \mathrm{C}$ eine Steigung von 1,4 und bei $37^{\circ} \mathrm{C}$ eine Steigung von 4,0 aufweist (Abb. 2 b).

\section{Diskussion}

Das überraschende Ergebnis der Untersuchung ist der Anstieg des kritischen Druckes und des Halbwertsdruckes oberhalb einer bestimmten maximalen $\mathrm{O}_{2}$-Aufnahme. Die Tab. 1 zeigt, daß für dieses Ergebnis nicht nur die Einstellzeit des polarographischen Meßsystems verantwortlich gemacht werden kann. Es ist deshalb zu diskutieren, welche Faktoren für dieses Phänomen entscheidend sein könnten:

1. In der atmenden Partikelsuspension könnte ein Inhibitor (z. B. Fumarat) wirksam werden. Wir haben bei mehreren Versuchen den $\mathrm{O}_{2}$-Druck bereits vor dem Start auf etwa 20 Torr gesenkt (durch Äquilibrierung mit $\mathrm{O}_{2} \mathrm{~N}_{2}$-Gemischen). Bei diesen Versuchen konnte also ein Inhibitor noch nicht in nennenswerten Konzentrationen vorhanden sein. Diese Versuchsanordnung hatte weiter den Vorteil einer erheblichen Verkürzung der Versuchsdauer und damit einer geringeren Schädigungsmöglichkeit der Sarkosomen. Diese Experimente ergaben die gleichen Ergebnisse wie unsere Normalansätze, welche bei Versuchsbeginn unter dem $\mathrm{O}_{2}$-Druck atmosphärischer Luft standen.

2. Die Sarkosomen-Suspensionen könnten trotz sorgfältiger Präparation Hämoglobin oder Myoglobin enthalten. In diesem Falle würde der Versuchsansatz einen $\mathrm{O}_{2}$-Speicher enthalten. Würde im Ansatz gleichzeitig ein druckunabhängiger $\mathrm{O}_{2}$-verbrauchender Prozeß stattfinden, dann ließe sich der Druckabfall in der Suspension wie folgt beschreiben: (Der $\mathrm{O}_{2}$-Speicher möge sich entlang einer L a n g m u i r schen Adsorptionsisotherme entladen.) 


$$
p=\frac{p_{1}}{v_{\mathrm{a}}} \cdot\left(V_{a}-Q \cdot t+\frac{a \cdot p_{1}}{K+p_{1}}-\frac{a \cdot p}{K+p}\right) .
$$

$p=$ Sauerstoffdruck zur Zeit $t$ (Torr),

$Q=$ Geschwindigkeit des $\mathrm{O}_{2}$-verbrauchenden Vorganges $\left(\mu \mathrm{l} \mathrm{O}_{2} / \mathrm{min}\right)$,

$a=$ Sauerstoffkapazität des Speichers $\left(\mu \mathrm{l} \mathrm{O}_{2}\right)$,

$K=$ Halbsättigungsdruck des Speichers (Torr),

$V_{\mathrm{a}}=$ Anfangsvolumen des physikalisch gelösten $\mathrm{O}_{2}$ $(\mu \mathrm{l})$ beim $\mathrm{O}_{2}$-Druck $p_{1}$.

Setzt man $Q^{\prime} / Q\left(Q^{\prime}=\right.$ gemessene Geschwindigkeit $)$ $=n$, so ergibt sich:

$$
n=\frac{V_{\mathrm{a}} \cdot(K+p)^{2}}{V_{\mathrm{a}} \cdot(K+p)^{2}+K \cdot a \cdot p_{1}} .
$$

Für $V_{\mathrm{a}}=10 \mu \mathrm{l} \mathrm{O}_{2}, p_{1}=150$ Torr, $K=20$ Torr und $a=0,03 \mu \mathrm{l} \mathrm{O}$ ergibt sich $n=0,99$ für 10 Torr. Gibt man für die Meßapparatur einen Fehler von $1 \%$ vor, so erschiene die Atmung nur bis etwa 10 Torr vom $\mathrm{O}_{2}$-Druck unabhängig. Das heißt, auch kleinste Mengen Myoglobin und Hämoglobin können die Meßergebnisse verfälschen.

3 . Bei der von uns verwendeten polarographischen Meßanordnung befand sich kein Gasraum über dem Suspensionsmedium. Demnach konnte nur der Gasaustausch zwischen den atmenden Partikeln und dem umgebenden Medium limitierend sein. Langmuir und $\mathrm{MCCABE}^{7}$ haben diese Möglichkeit unter Benutzung der von Rashevsky ${ }^{8}$ und Sullivan ${ }^{9}$ angegebenen Funktionen theoretisch berechnet. Nimmt man an, daß die Atmung der Sarkosomen vom $\mathrm{O}_{2}$ Druck unabhängig ist, so gilt für den Idealfall kugeliger Teilchen nach Rashevsky ${ }^{8}$ in einer gerührten Suspension:

$$
\begin{gathered}
c_{0}=c^{+}\left(1-n \cdot y^{2}-y^{3}+n \cdot y^{3}\right) . \\
n=\frac{A \cdot r_{0}{ }^{2}}{2 \cdot D_{\mathrm{i}} \cdot c^{+}} .
\end{gathered}
$$

$c_{0}=\mathrm{O}_{2}$-Konzentration in der Umgebung des Teilchens,

$c^{+}=$kritische $\mathrm{O}_{2}$-Konzentration,

$D_{\mathrm{i}}=$ Diffusionskoeffizient im Inneren des Teilchens.

$$
y=\frac{v_{\max }-v}{v_{\max }} .
$$

$r_{0}=$ Radius des Teilchens.

(Für $n$ gilt: $0 \leqq n \leqq 3$; ist $n$ kleiner als 3 , so ist ein meßbarer Einfluß der Membranpermeabilität vorhanden.)

7 I. S. Langmuir u. M. McCabe, J. theoret. Biol. 8, 124 [1965].

8 N. Rashevsky, Mathematical Biophysics physico-mathematical foundation of biology. N. Y., Dover Publications, 3rd Edition, New York 1960.
Die Abhängigkeit des $\mathrm{O}_{2}$-Verbrauches atmender kugeliger Teilchen ist in Abb. $2 \mathrm{a}$ in Abhängigkeit von $n$ dargestellt. Man erkennt deutlich die formale Ähnlichkeit der eingezeichneten Ergebnisse für $22,5{ }^{\circ} \mathrm{C}$ in Tris-Puffer mit der berechneten Kurve unter der Bedingung, daß $n=3$ ist. In diesem Fall ergab sich kein Einfluß der Membranpermeabilität.

4. Ist die Atmung kugeliger Teilchen vom $\mathrm{O}_{2}$ Druck abhängig, so kann man nach dem Vorschlag von Langmuir und $\mathrm{McCabe}^{7}$ die von Sullivan ${ }^{9}$ angegebenen Funktionen zur Analyse der Meßwerte verwenden. Nach Sullivan gilt

$$
\frac{p}{v} \sim \frac{K_{\mathrm{m}}}{v_{\max }-v}+W .
$$

$\left(K_{\mathrm{m}}=\mathrm{M}\right.$ ich a el is - Konstante; $W$ ist eine Funktion des Radius, der Membranpermeabilität und des Diffusionskoeffizienten.) Wir haben unsere Ergebnisse in einem Koordinationssystem

$$
\left(\frac{p}{v} \operatorname{gegen} \frac{1}{v_{\max }-v}\right)
$$

aufgetragen und dabei festgestellt, daß die Herzmuskelsarkosomen in Phosphatpuffer keinen Einfluß des verminderten $\mathrm{O}_{2}$-Druckes auf die Atmung erkennen lassen. Der kritische $\mathrm{O}_{2}$-Druck müßte deswegen wesentlich niedriger als 0,3 Torr (bei $30{ }^{\circ} \mathrm{C}$ und in Phosphatpuffer) liegen. Diese Ergebnisse stimmen mit den an Herzmuskelsarkosomen ermittelten Werten von Schindere und Mitarb. (zitiert nach Chance ${ }^{10}$ ) überein. Chance gab 1965 kritische Drucke von etwa 0,05-01,10 Torr an. LüBbers und Kessler ${ }^{5}$ haben mit der polarographischen Methode für Lebermitochondrien kritische $\mathrm{O}_{2}$-Drucke von $3-4$ Torr $\left(37{ }^{\circ} \mathrm{C}\right)$ gefunden. Es liegt der Schluß nahe, daß unter in vivoBedingungen der kritische $\mathrm{O}_{2}$-Druck durchaus in $\mathrm{Be}$ reichen unter 1 Torr liegen kann. Ob die von uns für $37{ }^{\circ} \mathrm{C}$ (Tris-Puffer) gefundene sigmoidförmige Abhängigkeit der Atmung vom $\mathrm{O}_{2}$-Druck den Verhältnissen in der Herzmuskelzelle entspricht, ob der Exponent $r=4$ ein $\mathrm{Ma}$ für die Stöchiometrie der Reaktion des $\mathrm{O}_{2}$ darstellt und ob vielleicht ein allosterischer Effekt vorliegt, muß vorläufig offen bleiben.

9 D. G. O'Sullivan, J. theoret. Biol. 2, 117 [1962].

10 B. Chance, J. gen. Physiol. 49, 163 [1965]. 